\title{
Intersubunit communications within KaiC hexamers contribute the robust rhythmicity of the cyanobacterial circadian clock
}

\author{
Yohko Kitayama ${ }^{1, *}$, Taeko Nishiwaki-Ohkawa ${ }^{1,2}$ and Takao Kondo ${ }^{1}$ \\ ${ }^{1}$ Division of Biological Science, Graduate School of Science, Nagoya University and CREST, Japan Science and Technology Agency \\ (JST), Furo-cho, Chikusa-ku, Nagoya 464-8602, Japan. \\ 2 Present address: Institute of Transformative Bio-Molecules (WPI-ITbM), Nagoya University, Furo-cho, Chikusa-ku, Nagoya 464-8602, \\ Japan. \\ * Corresponding Author: Dr. Yohko Kitayama, Division of Biological Science, Graduate School of Science, Nagoya University, Furo-cho, \\ Chikusa-ku; Nagoya 464-8602, Japan; Tel.: +81-52-789-2495; Email: kitayama@bio.nagoya-u.ac.jp
}

Circadian rhythms, endogenous oscillations with periods of $\sim 24 \mathrm{~h}$, are found in many organisms, and they enhance fitness in alternating day/night environments. In cyanobacteria, three clock proteins, KaiA, KaiB, and KaiC, control the timekeeping mechanism. KaiC, the central component of the system, is a hexameric ATPase that also has autokinase and autophosphatase activities. It has been assumed that KaiC's hexameric structure was critical for regulation of the circadian clock; however, the underlying molecular mechanism of such regulation has remained unclear. Recently, we elucidated the regulation of KaiC's activities by its phosphorylation state, in the context of its hexameric structure. We found that local interactions at subunit interfaces regulate KaiC's activities by coupling the nucleotide-binding states. We also discovered the mechanism of regulation by intersubunit communication in KaiC hexamers. Our observations suggest that intersubunit communication precisely synchronizes KaiC subunits to avoid dephasing, and contributes to the robustness of circadian rhythms in cyanobacteria [Kitayama, Y. et al. Nat. Commun. 4:2897 doi: 10.1038/ncomms3897 (2013)].

In a wide range of organisms, the circadian clock coordinates various biological activities in order to enable adaptation to daily environmental changes. Damage to the circadian clock can affect many biological activities. Cyanobacteria are the simplest organisms known to have a circadian clock. In the cyanobacterium Synechococcus elongatus
PCC7942, the KaiA, KaiB, and KaiC proteins are essential for generation of the circadian rhythm. When KaiA, KaiB, KaiC, and ATP are mixed in a test tube, the phosphorylation state of KaiC exhibits a robust circadian rhythm (Figure 1A). The successful in vitro reconstitution of this system indicates that the biochemical properties of clock proteins are the basis of the circadian clock. Previously, it was believed that the mechanism responsible for eukaryotic circadian oscillations is a transcriptional-translational feedback loop, in which clock gene products repress their own transcription. Recently, however protein-based circadian oscillations have been revealed to be ubiquitous in all domains of life. To date, the cyanobacterial clock is the sole circadian system to have been reconstituted in vitro, and it is therefore expected to be an excellent model for understanding the principles underlying biochemical circadian oscillators.

KaiC has ATPase, autokinase, and autophosphatase activities, all of which are crucial for circadian rhythmicity. KaiC is phosphorylated at serine 431 (S431) and threonine 432 (T432); this phosphorylation is modulated by the phosphorylation state of KaiC itself and by KaiC's phosphorylation state-dependent interactions with KaiA and KaiB. These regulatory interactions are thought to be basis of the phosphorylation cycle of KaiC. Rhythmic changes of KaiC phosphorylation state cause rhythmic gene expression in vivo. Thus, regulatory mechanism of KaiC activities is a basis of circadian clock. KaiC is a ring-shaped homohexamer, and it has been assumed that the KaiC's activities are regulated as hexamer. However, the mechanism by which these three activities are regulated and the functional importance of the hexameric structure in determin-

MICROREVIEW on: Yohko Kitayama, Taeko Nishiwaki-Ohkawa, Yukiko Sugisawa and Takao Kondo (2013). KaiC intersubunit communication facilitates robustness of circadian rhythms in cyanobacteria. Nat. Commun. 4:2897. doi: 10.1038/ncomms3897 
ing circadian rhythm have not been experimentally demonstrated.

To identify these regulatory mechanisms, and determine the influence of interactions at the subunit interface on KaiC's activities, we reconstituted KaiC hexamers containing different variant protomers and measured their autophosphatase and autokinase activities. We found that KaiC autodephosphorylation and autophosphorylation occur at subunit interfaces. ATP or ADP bind at each subunit interface, and the autophosphorylation sites face the nucleotide molecules on the neighboring protomers. We showed that there is a correlation between the autophosphatase activity of KaiC and steady-state ADP binding, and that the phosphorylation states of KaiC modulate nucleotide-binding state of Kaic. These observations indicate that KaiC's autokinase and autophosphatase activities are regulated by phophorylation state by coupling the nucleotidebinding states (Figure 1B).

Because the KaiC hexamer has six reaction units at the subunit interfaces, it is conceivable that phosphorylation reactions are could be regulated by interactions within hexamers, as well as by the adjacent protomer. Therefore, we next examined the effect of the phosphorylation states of the protomers on the overall activity of KaiC hexamers.

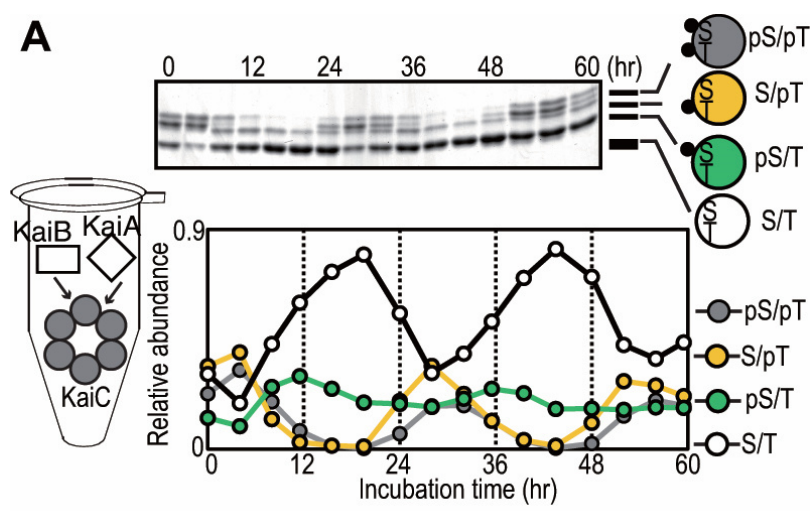

B
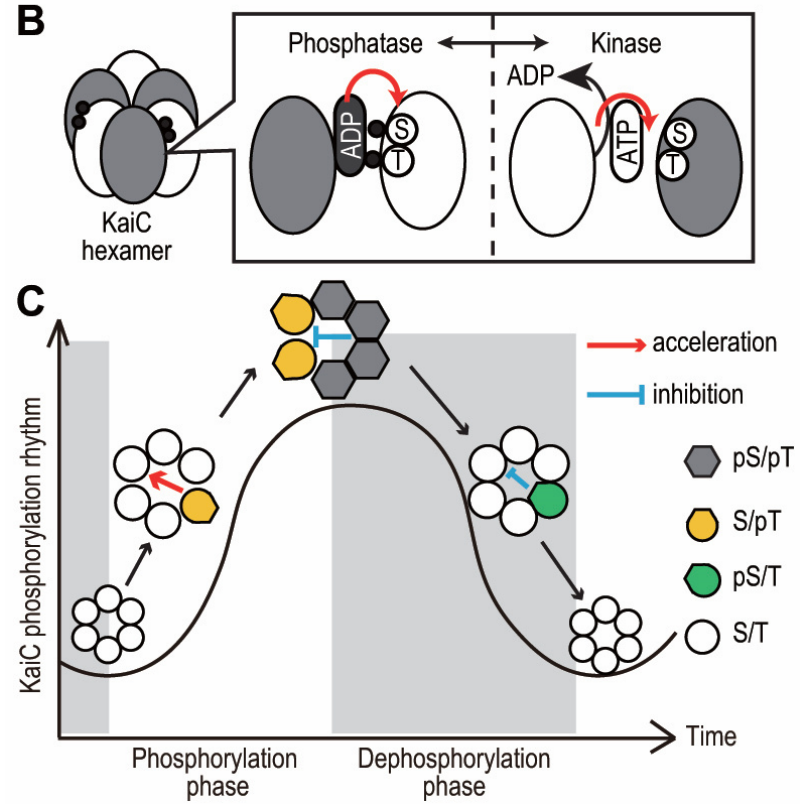

In the KaiC phosphorylation cycle, the phosphorylation proceeds in an ordered pattern. Unphosphorylated KaiC $(\mathrm{S} / \mathrm{T})$ is first phosphorylated at T432 (S/pT), which is further phosphorylated to the doubly phosphorylated form (pS/pT). The $\mathrm{T432}$ residue of $\mathrm{pS} / \mathrm{pT}$ is dephosphorylated first, yielding KaiC phosphorylated only at S431 (pS/T) (Figure 1A). We prepared reconstituted hexamers from 1:1 mixtures of KaiC-WT and four phosphorylation-site mutants that mimic each phosphorylation state in the KaiC phosphorylation cycle. Kinase activity was dependent on the specific combination of WT and mutant proteins, demonstrating the existence of intersubunit communications. T432phosphorylated protomers, corresponding to KaiC in the phosphorylation phase, enhanced the kinase activity of other protomers in the same hexamer; inversely, S431phosphorylated protomers corresponding to KaiC in the dephosphorylation phase decreased kinase activity. To determine whether intersubunit interactions are important for circadian rhythms, we incubated reconstituted hexamers from a 5:1 mixture of wild-type and phosphorylation-mutant monomers, and then incubated these hexamers with KaiA and KaiB to monitor the phosphorylation states of KaiC-WT. As a result, the circadian rhythmic of KaiC phosphorylation was lost. These observations suggest

FIGURE 1: Model of the regulation of the KaiC phosphorylation cycle within KaiC hexamer. (A) In vitro oscillation of KaiC phosphorylation. KaiC were incubated with KaiA and KaiB. Aliquots of the reaction mixtures were collected, and then subjected to SDSPAGE and Coomassie Brilliant Blue staining. From most to least mobile, bands represent the $\mathrm{S} / \mathrm{T}, \mathrm{pS} / \mathrm{T}, \mathrm{S} / \mathrm{pT}$, and $\mathrm{pS} / \mathrm{pT}$ forms of KaiC. The ratio of the amount of each form of KaiC to the total amount of KaiC is plotted against the incubation time. The phosphorylation proceeds in an ordered pattern, as follows: $\mathrm{S} / \mathrm{T} \rightarrow \mathrm{S} / \mathrm{pT} \rightarrow \mathrm{pS} / \mathrm{pT} \rightarrow \mathrm{pS} / \mathrm{T}$. (B) Schematic model of regulation at subunit interfaces. Phosphorylation takes place at subunit interfaces in KaiC hexamers. The phosphorylation states at two phosphorylation sites regulate the nucleotide-binding state at the subunit interface. ADP at the active site promotes dephosphorylation, and ATP at the active site promotes phosphorylation. (C) Schematic model of regulation by intersubunit communications. KaiC in the phosphorylation phase enhances the kinase activity of other protomers in the same hexamer; inversely, KaiC in the dephosphorylation phase decreases kinase activity. This intersubunit communication allows KaiC molecules that were phosphorylated in the wrong order to turn back, while allowing molecules in the appropriate phosphorylation state to move forward through the cycle. As a result, phase coherence among the ensemble of phosphorylating KaiC hexamers is achieved. 
that intra-hexameric interactions synchronize the phosphorylation reaction direction, resulting in stable and robust KaiC phosphorylation rhythm (Figure 1C).

Cyanobacteria live in a fluctuating environment: light and temperature change on a daily cycle, and these changes in turn affect transcription, translation, and cellular metabolism. Even in such a dynamic environment, however, the circadian clock continues to oscillate. The resilience of the KaiC phosphorylation rhythm is likely to be a source of the robustness of the oscillation in living cells. Intersubunit communication could precisely synchronize KaiC subunits in order to avoid dephasing, thereby facilitating robust circadian rhythms in cyanobacteria.

In light of these studies, several important questions arise. First, the relationship between the autoregulatory mechanism of KaiC and important properties of circadian clock such as temperature compensation of circadian period, remain to be clarified. Second, how can the phosphorylation state of one protomer regulate kinase activity throughout the whole hexamer? The potential roles of interactions between residues at the subunit interface, or the flexibility/rigidity of the KaiC ring, should be investigated. In addition, the relationship between the autoregulatory mechanism of KaiC and KaiA/KaiB, which are regulators of KaiC's activities, remain to be characterized. Finally, future studies should attempt to determine the functional significance of KaiC intersubunit communication in living cells. Robustness of oscillation in the context of molecular noise is a general and important feature of circadian clocks. Clarification of these issues will accelerate our understanding of the principles underlying these clocks.

\section{ACKNOWLEDGMENTS}

This work was supported in part by Grants-in-Aid, 24770043 (to Y. K.), 23118708 (to T.N.), and 24000016 (to T.K.) from the Ministry of Education, Culture, Sports, Science and Technology of Japan.

\section{CONFLICT OF INTEREST}

The authors declare no competing financial interests.

\section{COPYRIGHT}

(C) 2014 Kitayama et al. This is an open-access article released under the terms of the Creative Commons Attribution-NonCommercial-NonDerivative 3.0 license, which allows readers to download the article and share it with others, provided that the original authors and source are acknowledged. The article cannot be changed in any way or used commercially.

Please cite this article as: Yohko Kitayama, Taeko NishiwakiOhkawa and Takao Kondo (2014). Intersubunit communications within KaiC hexamers contribute the robust rhythmicity of the cyanobacterial circadian clock. Microbial Cell 1(2): 67-69. doi: 10.15698/mic2014.01.129 